\title{
Research on Observation Method of Building Displacement Based on Special Environment
}

\author{
Zipan Zhang ${ }^{1 *}$, Lei Wang ${ }^{2}$, Wenpeng Li $^{1}$, Pengpeng Zhu ${ }^{1}$, Hengle Cui ${ }^{1}$ \\ ${ }^{1}$ Shandong Zhongji Geographic Information Technology Co.LTD. Jinan 250000 China \\ ${ }^{2}$ Shandong Weifang Construction Group Co. LTD. Weifang 261000 China
}

\begin{abstract}
In some building construction, due to the influence of many factors such as site environment, geological conditions, construction design plan. the construction site environment is complicated and the construction process is staggered. For deformation monitoring under such conditions, a single, conventional monitoring method (baseline method, direction intersection method, corner measurement, etc.) may be difficult to carry out. If according to the characteristics of the project, several monitoring methods are used for comprehensive monitoring. It can better solve the difficult problem of on-site monitoring, and can significantly improve operation efficiency. This paper takes construction monitoring as an example to discuss the research of building displacement observation method under special environment.
\end{abstract}

\section{Introduction}

With the rapid development of my country's economy and the rapid development of modernization, more and more high-rise buildings are being built in large and mediumsized cities. Since these buildings need to be excavated with a deeper foundation (about $30 \mathrm{~m}$ ) during construction, a large foundation pit or high-cut slope will be formed after the foundation excavation, which will make it adjacent to the foundation of the building, especially the high-rise building Deformation will affect its normal use. In severe cases, it will cause landslides and endanger people's lives and properties[1-3]. Therefore, it is very necessary to observe the deformation of the building and its foundation during the construction period. Deformation monitoring of buildings and their foundations has a complete set of theoretical methods, and has also accumulated rich experience (for example, in June 1985, the accurate prediction and forecast of the Three Gorges landslide of the Yangtze River saved more than 10,000 lives), But there are also blood lessons[4-5]. These have made mankind more clearly realize the importance of deformation monitoring of high-rise buildings and their foundations. To this end, the state allocates special funds to deal with the mountains along the western river landslide section. The monitoring and observation of the movement and deformation of highrise buildings, important structures and their foundations in large and medium-sized cities has been put on the agenda. For urban built-up areas, especially in mountain cities (such as Chongqing and Guiyang)[6-8].There are large fluctuations in height, tall buildings, and poor visibility. If the traditional measurement method is used for daily deformation observation, it will bring great difficulties to the implementation of deformation monitoring, or even work. In actual work, we often care about the horizontal movement of buildings or slopes in important directions. In the basic horizontal movement monitoring of the teaching building of Chongqing No. 25 Middle School, we used the total station to measure the distance, carry out the distance difference correction, and find the horizontal movement value of the high-rise building foundation in important directions, which solved this problem[9-11].

\section{Building engineering characteristics and selection of monitoring methods}

The project is a second-class hotel building, divided into two buildings on the ground, east and west, and connected underground. The ancient temple is planned to be rebuilt between the east and west buildings. The main structure is 2 floors underground and 5 floors above ground. The building height is $12 \mathrm{~m}$. It is a reinforced concrete frame structure.

Since the project is relatively close to existing buildings (the nearest $5 \mathrm{~m}$ ), and there is an ancient building in the excavation area (the ancient building is an ancient temple and is an ancient cultural relic), it is protected by the Cultural Heritage Bureau, and it is planned to be after this construction To repair it, the foundation and pillars must not be damaged; the ancient trees on the west side of the site are also protected. Due to the limitations and influences of ancient trees, ancient buildings and surrounding existing buildings (too close) inside the site, the design requires that the construction be carried out according to the reverse excavation method. In order to ensure the smooth progress of construction, it is required to monitor the construction buildings and existing ancient temples. The monitoring content is as follows:

\footnotetext{
*Corresponding author: sdzjzhangzp@163.com, zjzzp@sdzjdlxxkj.cn
} 
(1) Monitoring the settlement of the foundation diaphragm wall;

(2) Settlement monitoring of the main structure of the project;

(3) Monitoring the horizontal displacement of the foundation continuous wall;

(4) Horizontal displacement monitoring of ancient buildings;

(5) Monitoring of settlement of ancient buildings.

According to the various deformation limits proposed by the design, the monitoring warning value is determined, referring to the experience of similar projects in the past, and $70 \%$ of the various deformation limits are used as the warning value. The specific values are shown in Figure 1.

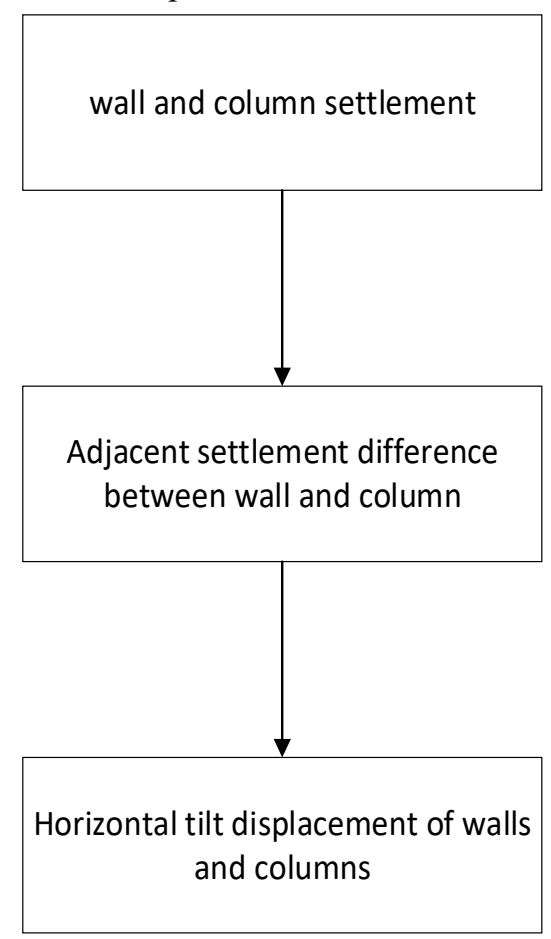

Fig1. structure diagram

The monitoring content is divided into two parts: horizontal displacement and vertical settlement; monitoring objects include existing buildings and buildings under construction; engineering construction adopts top-down excavation method, the site is small and the visibility is difficult, which is very unfavorable to the development of monitoring work. Settlement monitoring is generally less affected by site conditions and construction processes. Settlement observation points can be buried on the foundation diaphragm, ancient building pillars and the main body of the building under construction, and the measurement shall be carried out according to the secondclass technical requirements. The conventional methods of horizontal displacement monitoring generally use baseline method, direction intersection method, edge angle measurement and other monitoring methods[1]. The above methods generally first bury fixed observation signs outside the site, and use theodolite or total station to observe the subject to be monitored and the fixed Observe the changes in the relationship between the edges and corners of the signs to analyze the horizontal displacement of the subject to be monitored. However, due to the narrow site of the survey area, the project

Small, top-down method construction, the construction process is staggered, and the conventional and single monitoring method is very unfavorable to the development of monitoring operations. In order to better solve the above problems, we use an inclinometer to measure the horizontal displacement of the foundation continuous wall, and the vertical method to measure the horizontal displacement of the ancient building column.

The settlement observation datum point is the datum of settlement deformation observation. In order to ensure the accuracy of observation, 3 deep pile working foundation points were set up outside the settlement area far from the affected area of the construction area, and the deep pile point buried depth was about $20.0-25.0 \mathrm{~m}$. The hole is constructed with a 100-type water well drilling rig, and then steel bars are inserted into the hole. The center of the top is ground into a ball as a level mark. The lower part of the hole is poured with concrete, and the upper part is filled with fine sand to ensure the solid and stable reference control point. This constitutes an elevation reference control monitoring network, which serves as a reference control for settlement observation. The subsidence observation datum control network is observed in a closed leveling route according to the technical requirements of first-class leveling.

For the settlement observation of ancient buildings, basic diaphragm walls and buildings to be built, the layout principles and observation methods of their points are the same. The point density is required to be moderate, and the overall and local deformation of the building can be monitored. In view of the geological conditions and structural characteristics of the project, the layout method is as follows: nail steel nails at the lower end of the ancient building's column as a settlement observation point; at the top of the continuous wall crown beam every $15-20 \mathrm{~m}$ or at the main corner feature part Set up settlement observation points at locations, and appropriately dense observation points at ancient trees and ancient buildings; set up settlement observation points on both sides of the main column of the underground garage and the post-settlement pouring zone.

Before the earth excavation after the crown beam top of the continuous wall is completed, the horizontal displacement of the continuous wall is measured. Use CXE 03 type inclinometer for monitoring, the monitoring accuracy can reach $0.2 \mathrm{~mm} / 0.5 \mathrm{~m}$. First of all ,Connect the probe of the inclinometer and the reader through the connecting cable, and check whether the inclinometer tube and the inclinometer are working properly. Gently insert the probe into the inclinometer tube (the guide wheel is correctly inserted into a pair of guide grooves), start from the bottom of the hole, and read every $2.0 \mathrm{~m}$ along the entire length of the guide groove from the bottom to the top, and the display automatically records until it reaches At the top of the inclinometer tube, the probe should be stabilized in a certain position each time it is measured. After the measurement is completed, the probe should be lifted and rotated $180^{\circ}$ to reinsert the same pair of guide grooves to repeat the measurement. The two readings should be close in value and opposite in sign. 


\section{Building settlement observation method}

Measure the amount of deformation of the building and its foundation under the load of the building itself or under the action of external forces, and the analysis and processing of its data. The content includes settlement, tilt, displacement, deflection, wind vibration, etc. Deformation observation project. Its purpose is to monitor the safety of the building during construction and after it is completed; it is to verify the reliability of geological survey data and design data; to study the causes and laws of deformation to improve design theory and construction methods .

Deformation observation of building foundation and foundation mainly includes:

(1) Rebound measurement of foundation pit. Before, during and after the excavation of the foundation pit, the observation points buried in the foundation surface in advance are measured, and the elevation changes caused by the excavation of the foundation pit are measured. The elevation difference between the two times before and after the excavation is the total of the foundation pit Rebound amount.

(2) Layered settlement measurement of foundation. Measure the elevation changes of observation points buried in different soil layers due to the increase in load to obtain the settlement of each soil layer and the maximum depth of the pressure layer.Settlement measurement of buildingsMeasure the difference in elevation caused by the passage of time or changes in the foundation of the observation point on the building or foundation, and compare the observation values of different periods to obtain the settlement.

The above content belongs to the deformation observation based on vertical displacement. The method is to first arrange the deformation control network points reasonably according to the topography, geological conditions of the building site and the accuracy requirements of the deformation observation (see engineering control measurement). It is relatively stable near the building. The working base point is buried at the position to directly determine the displacement of the observation point on the building. As much as possible, the reference point (check point) is buried at a stable location outside the influence of deformation to check the stability of the working base point itself (see ground settlement (And horizontal displacement observation). The working base point and the reference point generally form a network, and are measured and inspected by precision leveling. The determination of the elevation change value usually uses a precision leveling method, and a hydrostatic level or bubble tilt meter can also be used, Electronic level, etc. for measurement.

The observation contents of the deformation of the upper part of the building mainly include:

(1) Oblique observation. Measure the vertical deviation of the top of the building due to the differential settlement of the foundation or the action of external forces. Generally, observation points are set at the top and wall foundation, and the relative displacement value is regularly observed, and the displacement of the top center point relative to the bottom center point can also be directly observed Value, and then calculate the slope of the building.

(2) Displacement observation. Determine the horizontal displacement of the building due to the influence of the lateral load. The establishment of the observation point depends on the engineering situation and the direction of the displacement.

(3) Crack observation. Measure the cracks in the wall of the building due to the partial uneven settlement of the foundation. Generally, observation signs are set on both sides of the crack, and the position change of the crack is regularly observed to obtain the size and direction of the crack.

(4) Deflection observation. Measure the degree of deflection of the building after it is stressed. Generally, the horizontal displacement value of the observation point at different heights relative to the bottom point is measured in the vertical plane of the building.

(5) Observation of swing and rotation. Measure the amount of swing and distortion of high-rise buildings and tall structures under external forces such as wind, earthquake, and sunlight.

\section{Conclusion}

For important buildings, the accuracy of horizontal movement and deformation observation under different complex environments is required to be higher (second level), but in large and medium cities, due to the dense buildings, it is impossible to measure with traditional methods. In order to solve this problem, an in-depth analysis was carried out, and the conclusions are as follows:

(1) Choose points to be flexible. The base point can be selected on a stable roof, cement ground or bedrock. The distance between the base point and the observation point can be close or far depending on the terrain.

(2) The observation method is simple. Only need to measure the distance and vertical angle from the base point to the observation point.

(3) High precision. It is close to the accuracy requirement of deformation measurement.

\section{References}

1. [1] coupling effect of tunnel blasting vibration and uneven settlement on buildings[J]. Engineering Construction, 2019, 51(07): 19-23.

2. Chen Kunxiang. Research on Building Deformation Monitoring Method Based on UAV[D]. Huazhong University of Science and Technology, 2019.

3. Xie Le. Study on the impact of deep foundation pit excavation of subway station on surrounding buildings [D]. Hefei University of Technology, 2019.

4. Wang Guoyu, Chen Guizhen, Kang Guangqing. Determination of observation accuracy and period in building deformation observation $[\mathrm{J}]$. Innovation in Science and Technology, 2019(05): 121-122.

5. Xin Yahui. Analysis of the influence of shield tunnel under the building force $[\mathrm{J}]$. Construction Technology, 2018, 47(S4): 1440-1444. 
6. Jin Hui. Discussion on the Displacement Monitoring Method of Rail Transit Buildings[J]. Gansu Science and Technology, 2018, 34(23): 95-96.

7. Hu Jiwei. The impact of underground comprehensive pipe gallery construction on ground settlement and adjacent buildings [D]. Xi'an University of Science and Technology, 2018.

8. Jiang Xiaoting. The impact of shield tunnel construction on special environments such as buildings [D]. Tianjin University, 2014.

9. Jiang Yitao. Understanding of the importance of equipotential testing of metal objects in special environments[J]. Electronic Technology and Software Engineering, 2013(23):151-152.

10. Jiang Yingjie, Sun Zhiqiang, Gong Erling, Xie Hongwei. Quantitative method of human error probability under probabilistic cognitive control mode[J]. Journal of National University of Defense Technology, 2011, 33(06): 175-178.

11. BS 8110-2-1985, Structural application of concrete. Code of practice for special environments $[\mathrm{S}]$. 